\title{
Proceeding
}

8th INSHS International Christmas Sport Scientific Conference, 5-7 December 2013. International Network of Sport and Health

Science. Szombathely, Hungary

\section{Differences in intensity of game load between senior and U19 female basketball players}

\author{
TOMÁŠ VENCÚRIK \\ Faculty of Sport Studies, Masaryk University, Brno, Czech Republic
}

\begin{abstract}
VENCÚRIK, T. Differences in intensity of game load between senior and U19 female basketball players. J. Hum. Sport Exerc., 9(Proc1), pp.S422-S428. The aim of this study is to compare the intensity of game load in individual playing positions between female basketball players in U19 (1st division) and senior (2nd division) categories. Eight female basketball players of U19 category and 9 female basketball players of senior category participated in this research. Individual intensity zones of the physical activity intensity were determined on the basis of the maximum heart rate (HRmax). HRmax was set in the endurance shuttle run test. The classification of the physical activity intensity: 1. $<50 \%$ of HRmax - very light intensity, $2.50-63 \%$ of HRmax - light intensity, 3. 64-76\% of HRmax - moderate intensity, 4. 77-93\% of HRmax - hard intensity, 5. 94-100\% of HRmax - very hard intensity. HR was during the game monitored with the system Suunto Team Pack. When the individual players' positions, in particular zones, were compared in the two categories, no significant differences were found - the point guard $(p>0.05)$, forward $(p>0.05)$ and center $(p>0.05)$. Moreover, no significant differences were detected, when the two categories were compared, in $\%$ of HRmax ( $p>0.05$ ) between point guards ( $86 \pm 2.5$ vs. $87.4 \pm 4.2$ ), forwards (89.7 \pm 2.1 vs. $84.7 \pm 3.6$ ) and centers $(87.5 \pm 2.7$ vs. $88.7 \pm 2.9)$. The results indicate high physiological demands on individual players' positions in both categories. Information of this character can be useful for basketball coaches and conditioning specialists. It is suggested that to increase the number of examined subjects and games ould benefit similar future researches. Key words: HEART RATE, PLAYING POSITIONS, ZONES OF PHYSICAL ACTIVITY INTENSITY.
\end{abstract}

Corresponding author. Faculty of Sport Studies, Masaryk University, Brno, Czech Republic.

E-mail: vencurik@mail.muni.cz

8th INSHS International Christmas Sport Scientific Conference, 5-7 December 2013. International Network of Sport and Health Science. Szombathely, Hungary.

JOURNAL OF HUMAN SPORT \& EXERCISE ISSN 1988-5202

(c) Faculty of Education. University of Alicante

doi:10.14198/jhse.2014.9.Proc1.28

VOLUME 9 | Proc1 | 2014 | S422 


\section{INTRODUCTION}

Basketball is one of the most popular team sports in the world and it comprises of specific structural and functional characteristics. Game performance is therefore affected by multiple factors (technical, tactical, psychical, conditional, and social) which form one complex unit. From the conditional standpoint basketball is characteristic for its intermittent load, whilst it is demanding in the aerobic and anaerobic area. (Mclnnes et al., 1995; Rodríguez-Alonso at al., 2003; Abdelkrim et al., 2007). These conclusions were reached by the authors of stated studies on the basis of monitoring of the heart rate $(\mathrm{HR})$, measuring the concentration of blood lactate and time-motion analysis. All three methods were used during competitive games in various age and gender categories. It is evident that the analysis of the intensity of motor load under the force of game conditions is gradually acquiring prominent meaning in today's modern era. The primary reason for conducting this analysis is to make the training process more effective and thus the transfer from training to game should be effective. HR is one of the most widely used physiological variables in conditioning area. According to Achten \& Jeukendrup (2003) the monitoring of HR is appropriate and objective method used to evaluate the intensity of physical activity. The HR can be measured repeatedly without any financial costs and is non-invasive (Gocentas et al. 2011). In sport games, including basketball, to monitor the HR is from the financial point of view more costly since in the game telemetric devices without wrist watches have to be used. These devices are able to transmit HR of several players simultaneously (the number of monitored players depends on the manufacturer of the specific device) in real time and the progress may be followed on the screen of laptop.

Authors of several research studies (Vaquera et al., 2008; Matthew \& Delextrat, 2009; Narazaki et al., 2009; Montgomery et al., 2010) analysed the intensity of the game load and they tried to specify the physiological demands on individual player's positions during competitive games. Vaquera et al. (2008) state in their study statistically significant differences in percentage of maximum heart rate (HRmax) between the point guards and forwards, as well as between point guards and centers.

When comparing forwards and centers they did not record any statistically significant differences. The intensity of game load may probably vary depending on the interruptions during the game (free throws, time-outs, etc.), substitutions and as Hoffman (2003) states it may also vary because of the chosen game tactics.

The aim of this work is to determine the differences in the intensity of the game load between the same player's positions of two different age categories-the U19 and senior-in women's basketball.

\section{MATERIAL AND METHODS}

Eight female basketball players in category U19 (1st division-the highest competition in Czech Republic) and nine players in senior category (2nd division-second highest competition in senior category in Czech Republic). The average age of the U19 group was $17.4 \pm 0.99$, body height $181 \pm 5.95 \mathrm{~cm}$, and body weight $63.6 \pm 5.76 \mathrm{~kg}$. The average age of the senior group was $20.6 \pm 2.94$, body height $178.4 \pm 5.5 \mathrm{~cm}$, and body weight $64.9 \pm 5.84 \mathrm{~kg}$. In the U19 category were recognized these standard player's positions: point guard $(n=1)$, forward $(n=3)$, center $(n=4)$, and in senior category were: point guard $(n=2)$, forward $(n=4)$, and center $(n=3)$.

The HR was monitored during one game in the U19 category and one game in the senior category with the use telemetric device Suunto Team Pack (Suunto Oy, Vantaa, Finland). These competitive games were 
recorded on camcorder Canon HG10 (Canon Inc., Tokyo, Japan). The receivers of the HR (Suunto Memory Belts) were synchronized with the camcorder and starting time of the game. The HR was recorded in two-second intervals. Transmission of the data from the receivers to the laptop was used the docking station Suunto. The acquired data were subsequently evaluated in respective software programme Suunto Training Manager. To interpret the results the figures of HR monitored during the total game time were used. Total game time is here defined as the time the subject was on the court, including short interruptions in play, time-outs and free throws (Abdelkrim et al. 2007; Matthew \& Delextrat, 2009). Into the total game time were not included short breaks between the quarters, half-time and time spent on bench.

The figures of the HRmax in endurance shuttle run test were used to determine five zones of physical activity intensity (Thompson et al., 2010). The data are expressed as mean \pm standard of deviance (SD). The distribution of normality of data was verified by Shapiro-Wilk test. On the basis of the distribution of normality data the Student t-test and non-parametric Mann-Whitney U-test were used. The average played time in 1st and 2nd zone, in all players' positions, was so short that no other statistical processing was necessary. The software Statistica 12 (StatSoft, Inc., Tulsa, USA) was used for complete statistical evaluation of the gained data. Statistical significance was set to $\alpha=0.05$.

Table 1. Classification of physical activity intensity (Thompson at al., 2010)

\begin{tabular}{|c|c|c|}
\hline Zone & $\mathrm{HR}_{\max }(\%)$ & Intensity \\
\hline 1 & $<50$ & very light intensity \\
\hline 2 & $50-63$ & $\begin{array}{l}\text { light intensity } \\
\text { moderate }\end{array}$ \\
\hline 3 & $64-76$ & intensity \\
\hline 4 & $77-93$ & $\begin{array}{l}\text { hard intensity } \\
\text { very hard }\end{array}$ \\
\hline 5 & $\geq 94$ & intensity \\
\hline
\end{tabular}

\section{RESULTS}

The mean HR during the total game time was in the senior category $87.9 \pm 3.6 \%$ from HRmax and in U19 category $87.5 \pm 2.8 \%$ from HRmax. The results of the HR show that the point guard of the U19 played in 3rd, 4th and 5th zone $24.3 \pm 33.4 \%, 60.7 \pm 29.2 \%$, and $13.4 \pm 17 \%$, respectively, from the total game time. The mean HR was $86 \pm 2.5 \%$ from HRmax. The senior point guards played in 3rd zone $8.5 \pm 6.3 \%$, in 4 th zone $67.4 \pm 22.7 \%$, and in 5th zone $23.2 \pm 25.2 \%$ from total game time with mean HR $87.4 \pm 4.2 \%$ from HRmax. When the acquired figures were compared no statistically significant differences $(p>0.05)$ were found (Figure 1).

The forwards (U19 vs. seniors) played in 3rd zone $3.9 \pm 1.3 \%$ vs. $12.5 \pm 4 \%$ form total game time. In 4th zone $60.6 \pm 26.6 \%$ vs. $73.2 \pm 6.8 \%$ and in 5 th zone $35.3 \pm 26.1 \%$ vs. $11.1 \pm 9.8 \%$ from total game time. The mean HR reached in U19 89.7\% and in seniors 84.7\% from HRmax. The comparison of the figures in individual zones and percentage from the HRmax were not statistically significant, $p>0.05$ (Figure 2).

The centers of U19 played in 3rd, 4th and 5th zone $7.9 \pm 5.8 \%, 67.1 \pm 23.8 \%$, and $23.1 \pm 21.6 \%$ from total game time, respectively. The centers in the senior category played $7.6 \pm 5.7 \%$ in 3rd zone, $58.9 \pm 12 \%$ in 
4th zone, and $31.8 \pm 11.4 \%$ in 5th zone. The mean HR in U19 was $87.5 \pm 2.7 \%$ from HRmax compared to $88.7 \pm 2.9 \%$ from HRmax in senior category. Between centers no statistically significant difference in individual zones and percentage from HRmax $(p>0.05)$ was recorded (Figure 3).

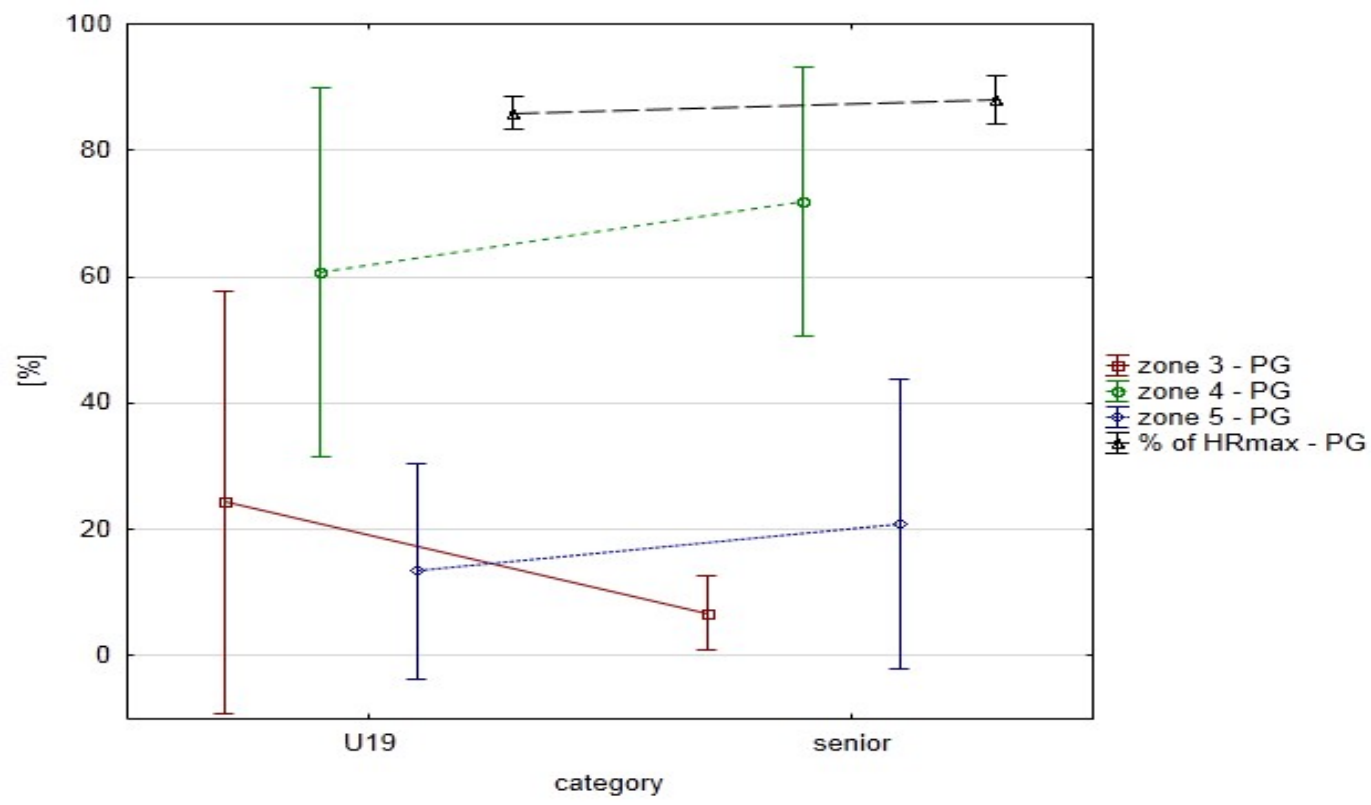

Figure 1. Comparison of point guards in categories U19 and senior

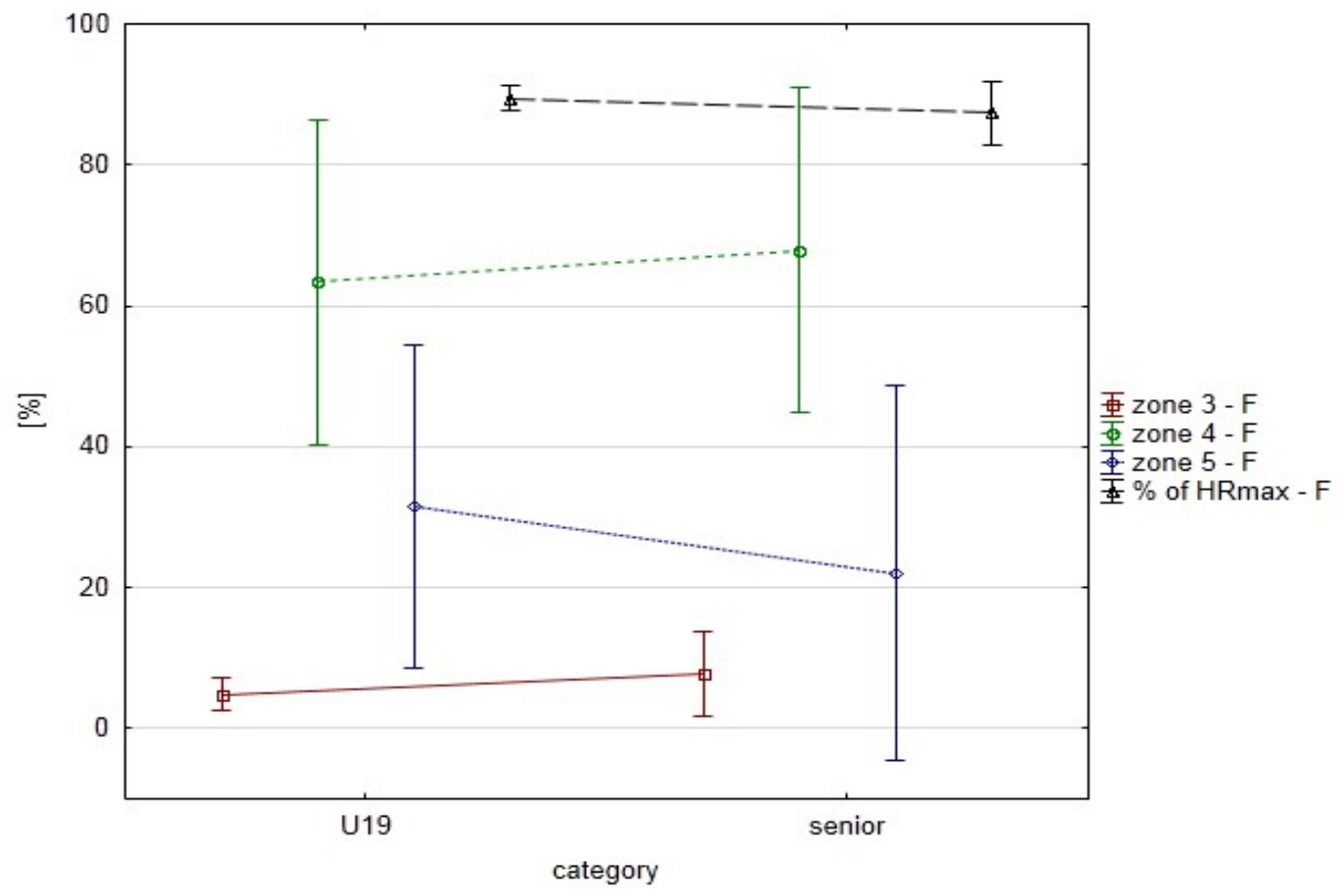

Figure 2. Comparison of forwards in categories U19 and senior 


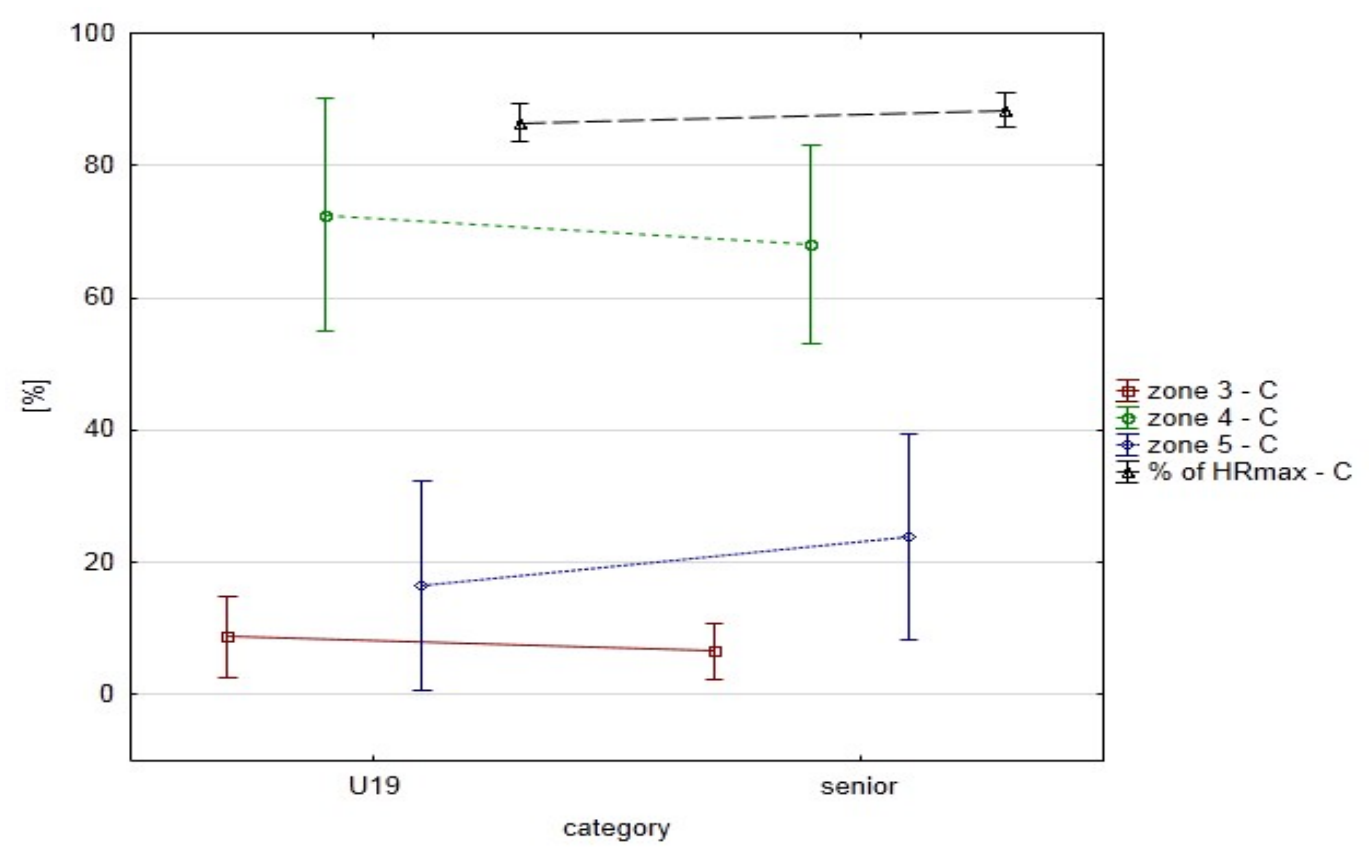

Figure 3. Comparison of centers in categories U19 and senior.

\section{DISCUSSION}

The HR values are sometimes very difficult to interpret and basketball game is not an exception. While interpreting the values of HR the suggestions of Ziv \& Lidor (2009) - Physical attributes, physiological characteristics... were consulted and presented results were introduced in percentage of HRmax. Moreover, the data was evaluated from the total game time because according to above mentioned authors live time ignores important information about the recovery of the players during the interruption of the game, as are free throws and time-outs. This study presents the comparison of game HR values in two different age categories of women's basketball—comparison of the same player's positions (point guard, forward and center) but in categories U19 (1st division) and seniors (2nd division). Forwards form U19 category played $95.9 \%$ of total time with HR (above $77 \%$ of HRmax) in 4th and 5th zone. In senior category the forwards played in 4 th and 5 th zone $84.3 \%$ of total time. Similar figures were reached by centers who played in U19 category $90.2 \%$ and senior category 90.7 of total game time in hard intensity and very hard intensity. Similar results were reached by Abdelkrim et al. (2010)-elite male players of U19 played $92.6 \%$ of total game time with HR higher than $75 \%$ of HRmax, $19.3 \%$ of total game time with HR higher than $95 \%$ which also resembles the presented results in this study (in this case figures above $93 \%$ of HRmax in range from $11.1 \%$ to $35.3 \%$ of total game time).

The mean HR values expressed as percentages of HRmax also correspond to the research of RodriguezAlonso et al. (2003), Abdelkrim et al. (2007), Vaquera at al. (2008), and Matthew \& Delextrat (2009). Rozdriguez-Alonso et al. (2003) state values of mean HR during national and international games of women's basketball- $-90.8 \%$ and $94.6 \%$ of HRmax, respectively.

Abdelkrim et al. (2007) state mean HR in U19 category of men's basketball on the level of $91 \%$ of HRmax. Vaquera et al. (2008) state HR of male point guards, forwards and centers at levels $95,6 \%, 93,7 \%$ a $92,6 \%$ of HRmax, respectively. 
In female senior category are the reached results comparable with the study of Matthew \& Delextrat (2009) where female bbasketball players played with mean HR $89.1 \%$ of HRmax. However, Scanlan et al. (2012) determined lower mean value of HR in state-level Australian women's competition-68.6\% of HRmax.

Data presented in this study and studies of above mentioned authors confirm the fact that physiological demands in basketball game may depend on several factors as gender, age group, level of competition, chosen tactics and others. Even if no statistically significant differences were found when the two age categories were compared, the values of HR show high physiological demands. HR may be also influenced by other factors like anxiety, psychological excitement and not only by the intensity and the duration of physical activity. Bangsbo (1994) assumes that the influence of psychological factors on the HR response may be lower during the physical activity in high intensity.

Game-based training seems as suitable choice when the results are transferred into the training process. The advantages of the game-based training are in stimulation of sport-specific movement patterns, development of technical and tactical skills in high physical load and better motivation of players (Gabbett et al., 2009). Sampaio et al. (2009) states similar values of HR in a game 3 on 3 ( $87 \pm 4 \%$ of HRmax) and in game 4 on 4 (83 $\pm 4 \%$ of HRmax). Klusemann et al. (2012) in their study they recorded level of mean HR $86 \pm 4 \%$ of HRmax in game 2 on 2 and $83 \pm 5 \%$ of HRmax in game 4 on 4 . Form physiological point of view these introduced values of HR (Sampaio et al., 2009; Klusemann at al., 2012) correspond to the values of $\mathrm{HR}$ presented in this study.

The results point out the high physiological demands on individual player's positions in both age categories. On the basis of the gained data it may be concluded that the transition form one category (the U19, 1st division) to the other (the senior, 2nd division) should be form the physiological point of view without complications. Nevertheless, we need to take into consideration also other factors (technical, tactical, psychological, social, etc.) that may influence the game performance. In the senior category of the 1st division, however, one should expect higher physiological (conditioning) demands.

\section{CONCLUSIONS}

This study compares player's positions in two different categories (U19 - 1st division and senior - 2nd division) from physiological point of view. The results indicate similar intensity of game load in both categories. The proposed information in this study may be useful for conditioning coaches and basketball coaches to manage and plan the training process (e.g. game-based training). Of course, this study has some limitations-more accurate results would have been reached if the number of researched subjects and competitive games was higher. If the blood lactate concentration was measured and time-motion analysis was used the compact view on the assessment of the game load intensity would be provided.

\section{REFERENCES}

1. Abdelkrim, N.B., Castagna, C., Jabri, I., Battikh, T., Fazaa, S.E. \& ATI, J.E. (2010). Activity profile and physiological requirements of junior elite basketball players in relation to aerobic-anaerobic fitness. J Strength Cond Res, 24(9), pp.2330-2342.

2. Abdelkrim, N.B., Fazaa, S.E. \& Ati, J.E. (2007). Time-motion analysis and physiological data of elite under-19-year-old basketball players during competition. Brit J Sport Med, 41(2), pp.69-75. 
3. Achten, J. \& Jeukendrup, A.E. (2003). Heart Rate Monitoring: Applications and Limitations. Sports Medicine, 33(7), pp.517-538.

4. Bangsbo, J. (1994). Energy demands in competitive soccer. J Sport Sci, 12, pp.5-12.

5. Gabbett, T., Jenkins, D. \& Abernethy, B. (2009). Game-Based Training for Improving Skill and Physical Fitness in Team Sport Athletes. International Journal of Sports Science \& Coaching, 4(2), pp.273-283.

6. Gocentas, A., Landõr, A. \& Kriščiūnas, A. (2011). Heart rate recovery changes during competition period in high-level basketball players. Education. Physical training Sport, 1(80), pp.11-16.

7. Hoffman, J.R. (2003). Physiology of basketball. In McKEAG, D.B. (Ed.) Basketball. Handbook of sport medicine and science. Indianapolis: Blackwell Publishing. pp. 2-24.

8. Klusemann, M.J., Pyne, D.B., Foster, C. \& Drinkwater, E.J. (2012). Optimising technical skills and physical loading in small-sided basketball games. J Sport Sci, 30(14), pp.1463-1471.

9. Mcinnes, S.E., Carlson, J.S., Jones, C.J. \& MCkenna, M.J. (1995). The physiological load imposed on basketball players during competition. Journal of Sports Sciences, 13(5), pp.387-397.

10. Matthew, D. \& Delextrat, A. (2009). Heart rate, blood lactate concentration, and time-motion analysis of female basketball players during competition. J Sport Sci, 27(8), pp.813-821.

11. Montgomery, P.G., Pyne, D.B. \& Minahan, C.L. (2010). The Physical and Physiological Demands of Basketball Training and Competition. International Journal of Sports Physiology and Performance, 5, pp.75-86.

12. Narazaki, K.; Berg, K.; Stergiou, N. \& Chen, B. (2009). Physiological demands of competitive basketball. Scand J Med Sci Spor, 19(3), pp.425-432.

13. Rodríguez-Alonso, M., Fernández-García, B., Pérez-Landaluce, J. \& Terrados, N. (2003). Blood lactate and heart rate during national and international women's basketball. J Sport Med Phys Fit, 43(4), pp.432-436.

14. Sampaio, J., Abrantes, C. \& Leite, N. (2009). Power, heart rate and perceived exertion responses to $3 \times 3$ and $4 \times 4$ basketball small-sided games. J Sport Sci, 18, pp.463-467.

15. Scanlan, A.T., Dascombe, B.J., Reaburn, P. \& Dalbo, V.J. (2012). The physiological and activity demands experienced by Australian female basketball players during competition. Journal of Science and Medicine in Sport, 15, pp.341-347.

16. Schönfelder, M., Hinterseher, G., Peter, P. \& Spitzenpfeil, P. (2011). Scientific comparison of different online heart rate monitoring systems. International Journal of Telemedicine and Applications, 2011.

17. Thompson, W.R., Gordon, N.F. \& Pescatello, L.S. (Eds.). (2010). ACSM's Guidelines for Exercise Testing and Prescription (8th ed.). Baltimore, USA: American College of Sports Medicine.

18. Vaquera, A., Refoyo, I., Villa, J.G., Calleja, J., Rodríguez-Marroyo, J.A., García-López, J. \& Sampedro, J. (2008). Heart rate response to game-play in professional basketball players. J Hum Sport Exerc, 3(1), pp.1-9.

19. Ziv, G. \& Lidor, R. (2009). Physical Attributes, Physiological Characteristics, On-Court Performances and Nutritional Strategies of Female and Male Basketball Players. Sports Med, 39(7), pp.547-568. 